\title{
Who does physics? Understanding the composition of physicists through the lens of women of color and LGBTQ+ women physicists
}

\author{
Xandria R. Quichocho, Jessica Conn, Erin M. Schipull, and Eleanor W. Close \\ Physics Department, Texas State University, 601 University Dr. San Marcos, TX, 78666
}

\begin{abstract}
Historically, research on "underrepresented" students in physics is conducted primarily at Predominately White Institutions and has focused on two separate identities: gender or race. Because of this, the unique intersections experienced by Women of Color (WoC) has been largely ignored. In addition, research on the experiences of Lesbian, Gay, Bisexual, Transgender or Queer (LGBTQ+) physicists in a collegiate setting is virtually non-existent. This paper will present common themes in the idea of who does physics through the lens of WoC and female LGBTQ+ physicists currently or recently enrolled in a Physics Program at a large Hispanic Serving Institution. Participants engaged in semi-structured interviews and were asked questions about their perceptions of their physics environments, available support systems, and how they described a physicist. The analysis employs the theory of Intersectionality and examines the narratives through a critical race and queer lens to fully understand the lived experiences of our participants.
\end{abstract}

\footnotetext{
2019 PERC Proceedings edited by Cao, Wolf, and Bennett; Peer-reviewed, doi.org/10.1119/perc.2019.pr.Quichocho Published by the American Association of Physics Teachers under a Creative Commons Attribution 4.0 license. Further distribution must maintain attribution to the article's authors, cover page, and DOI.
} 


\section{INTRODUCTION}

The Physics Education Research (PER) literature on the concept of physics identity has been expanding in the past few years. However, research that has been conducted and published on students in particular has taken place at predominantly White institutions, and few such investigations have been conducted at Minority Serving Institutions (MSIs). Research has largely ignored the intersections of race and gender [1], with even fewer studies on students who identify as Lesbian, Gay, Bisexual, Transgender, Queer, or other sexual minority (LGBTQ+) [2].

The recent APS report on LGBT climate in Physics found that over a third of LGBT physicists who responded to their broadly-distributed survey were at risk for leaving their school or workplace [2]. The authors also note that they found no prior published studies on the experiences of LGBT people in physics, either in terms of general climate or measuring persistence in degree programs or professional pathways.

The research on women of color in physics has also expanded in the last few years, most notably with the publishing of Ong et al.'s 2011 paper "Inside the Double Bind: A Synthesis of Empirical Research on Undergraduate and Graduate Women of Color in Science, Technology, Engineering, and Math" [1], and Hyater-Adam et al. 's 2018 paper "Critical look at physics identity: An operationalized framework for examining race and physics identity" [3]. However, despite the increase in literature, very few studies solely focus on undergraduate women of color in physics.

The research presented in this paper is a response to this lack of research on women of color, LGBTQ+ women physicists, and those who embody the intersections of these identities. In keeping with the definitions put forth by our participants, in this study we define a physicist as someone who has a physics identity and engages in the practices of physics $[4,5]$. In order to increase access to physics for these students, we must record, recognize, and understand the unique experiences they have in their physics programs and the elements that support or undermine their continued participation. In this paper we examine how four physicists who exist in the intersections of minoritized race, gender, and sexuality describe the stereotypes that surround being a physicist, their interpretation of physics as a practice, and how both of these inform their perceptions of themselves and their peers.

\section{BACKGROUND}

\section{A. Intersectional physics identities and the double bind}

This study focuses on the lived experiences of women of color and LGBTQ + women physics majors who attend, or have recently attended, Texas State University, a large public Hispanic Serving Institution (HSI) in Central Texas.
We analyzed the narrative data using the construct of intersectionality.

Intersectionality can be described as a holistic examination of a person's multiple social identities as they exist in the environments they inhabit. A term coined by Kimberlé Crenshaw and informed by Black Feminist Theory, intersectionality informs identity research through its emphasis on studying systems of oppression and highlights the importance of considering intersecting social identities [6]. Crenshaw describes the construct as a metaphor that allows the researcher to understand multiple forms of inequality and inequity, and how those who exist in an intersection of multiple marginalized identities experience a compounding of disadvantages, or an experience "greater than the sum of racism and sexism" [6]. The construct also includes a critical perspective on race and gender that allows the researcher to analyze the society and culture from which the oppression stems.

Within the intersectionality construct is this idea of certain populations who are doubly bound by stereotypes regarding their identities. Ong et al. describe the bound states as "unique challenges [women of color face] as they simultaneously [experience] sexism and racism in their STEM careers" [1]. They expand on this double bind with the additional consideration of how homophobia in STEM affects those who identify as LGBTQ+ women.

Applying this construct to our analysis allows us to avoid labelling a single participant's narrative as generalizable to those who may share their multiple social identities and centers the lived experience of the individual themselves. It also allows us to examine the societal, institutional, and cultural impact the culture of STEM has on individuals who must navigate through it. For women of color and LGBTQ+ women physicists, intersectionality can help describe the simultaneous identity negotiations they must conduct to navigate traditionally sexist, racist, and homophobic environments [1,3,7-9]. We assume that alongside the participants' multiple social identities they simultaneously have a physics identity that is developed through their participation in their physics program $[4,5,10]$.

\section{B. Minority serving institutions}

As a direct response to the inequity, oppression, and racism present in higher education, Historically Black Colleges and Universities (HBCUs) were created to give Black and other racially marginalized students access to higher education. As racial minority student enrollment in universities increased, the idea of institutions that serve these populations began two grow. Minority Serving Institutions (MSIs) are instrumental to the undergraduate education of racial minorities in physics. About $40 \%$ of physics bachelor's degrees earned by Black students are earned at HBCUs and Black Serving Institutions, and $20 \%$ of those earned by Hispanic students are earned at Hispanic Serving Institutions [11]. In 2012 MSIs made up around seven 
percent of all higher education institutions and enrolled nearly $20 \%$ of all undergraduate students [12].

We propose that MSIs may have more institutional support available for physicists from racially marginalized groups. Texas State was designated as an HSI in 2011. The Physics Department enrolls 48\% racial/ethnic minority students, $28 \%$ women overall, and $14 \%$ women of color [13]. While the enrollment numbers do show a department that is comprised of majority white students, the significantly high percentage of racial minorities allows us to assume that students of color-and specifically women of color-are likely to find others who share their identities. Because of this, the women in the department work, collaborate, study, and socialize in a department community with a multitude of students of color, giving them a uniquely diverse physics learning environment.

\section{Cultural stereotypes physicists}

The confident, oftentimes arrogant, white male has become the icon of the competent scientist in Western culture [7]. Those like Albert Einstein, Sheldon Cooper, and Richard Feynman have reached a level of celebrity to have become household names, perpetuating the monolithic image of those who do and are able to do science despite the changes we see happening in the demographics of real physicists. Women of color, who have been entering the field of physics in increasing numbers for decades, are largely ignored in the culture surrounding scientific celebrity $[7,14]$. The existence of women of color who participate in a field that is still overwhelmingly populated by those who fit into the "arrogant white male" stereotype can be seen as an act of rebellion in itself. However, there exist many biases and challenges that build barriers to women of color entering the elusive physicist club.

The ability to gain authority and prestige in physics is currently inseparable from the myth that science is a purely objective, meritocratic, and universal study and is beyond the scope of context and culture [2,3-6]. There is a persistent belief that the practice of physics happens in this genderless, raceless, and therefore neutral space. However, attributing objectivity and neutrality to the stereotype of the physicist creates a narrative that objective and neutral are synonymous with cis-gender, heterosexual, white man. Identity research on marginalized populations in physics thereby becomes "a source of strength, community, and intellectual development" [15] for those who do not inhabit the majority space.

In the interviews conducted for this project we observed that participants were all aware of the cultural stereotypes surrounding the physicist title and were aware of their position as marginalized people in the field. In spite of this, the women we interviewed have been able to negotiate their lived experiences in physics with their lived experiences in the double bind and identify fully as physicists.

\section{METHODS}

Students who self-identified as both a racial minority and a woman in university forms, and as a declared Physics major, were identified through a list provided by the department. Those who identified as LGBTQ+ were found entirely through in-person social networks. We define an "in-person social network" as a set of personal relationships between students or faculty members. Both the first and third authors were students in the department when the interviews were conducted and had peer relationships with the interviewees that allowed for the disclosure of their LGBTQ+ identities.

Participants were asked to participate in a semistructured, clinical-style, hour-long interview conducted by the first author. The first author identifies as a Black, Chamorro, and bisexual cisgender woman; this may have had an effect on what the participants felt comfortable discussing during the interview. The remaining three authors all identify as White cisgender women. Two identify as heterosexual; one chose not to disclose her sexual orientation in this publication. All authors aided in developing the interview protocol and analyzing the data.

The interview protocol was designed to elicit a narrative about the participant's experiences in her Physics program through her interactions with peers, faculty, and those outside of physics. The interviews were video recorded and transcribed. In this paper we focus on the question "Can you describe a physicist?"

To date, six interviews have been conducted with participants at the undergraduate (4), Master's (1), and Doctoral (1) level. In the analysis section we will more closely examine the narratives of four women: Nadya, Frankie, Autumn, and Paige (pseudonyms). Nadya, Frankie, and Autumn were interviewed during their last semester in their undergraduate program and Paige during her last semester in her doctoral studies. In Table I below we outline how the women described their identities.

\section{INTERVIEW DATA AND ANALYSIS}

Analysis of the participants' narratives began with reading the video transcripts and watching the clips where the interviewer asked "Can you describe a physicist?" Questions leading up to this point in the interview focused primarily on the interviewee's interactions with peers and

TABLE I. Racial/ethnic, sexual orientation, and gender identities of women in study.

\begin{tabular}{ll}
\hline \hline \multicolumn{1}{c}{ Name } & \multicolumn{1}{c}{ Identities } \\
\hline Nadya & Hispanic \& White, heterosexual, cisgender \\
Frankie & Hispanic \& White, bisexual, cisgender \\
Autumn & White, bisexual, cisgender \\
Paige & White, lesbian, cisgender \\
\hline \hline
\end{tabular}

experiences within the department. The exact wording of the question changed over the course of the study. Nadya was asked, specifically, "Can you describe a typical physicist?" 
However, through analysis of Nadya's initial response (quoted in section B of part IV below), the authors concluded that the word "typical" prompted a general cultural understanding of who does physics, and did not lead to a full picture of how the interviewees thought about the term.

Intersectionality, critical race theory, and communities of practice theory informed the interview protocol and data analysis. Participants were asked how they labelled their social identities in their own words, and how, if at all, these identities were negotiated in the physics space. In communities of practice theory, "identity as negotiated experience" is a lens for understanding identity in practice [5]. In the analysis, the research team emphasized using the participant's language and the researchers were cognizant of how their own identities informed their interpretations. Using participants' exact language and interpretations in the analysis legitimizes these experiences as valued research data, an important aspect of intersectional and critical race research.

The analysis revealed three common themes shared between almost all of the women: recognition and personal interpretations of common stereotypes surrounding physicists, physics as a practice, and the recognition of self and others as physicists. In the following section, quotations in italics are questions asked by the interviewer.

\section{A. Recognition and Interpretation of Stereotypes}

When answering the question "Can you describe a physicist?" the women interviewed often included in their answers a recognition of the ambiguity of who a physicist is, or mentioned common stereotypes.

Autumn, who was interviewed during her last semester in the physics major, recognized the ambiguity in who is allowed to call themselves a physicist. As she reflected aloud, she questioned the necessity of having credentials or formal instruction in order to be a physicist:

"It's like, do you need to have a degree to be a physicist or do you need to just be in class learning to be a physicist, or could [you] even be a physicist without even ever taking any classes or having any credentials?"

The stereotypical physicist holds a degree in physics, granted after taking a number of formal physics classes. Autumn recognizes this, but still questions the idea. This act of questioning the necessity of formal training is in part tied to her ability to identify as a physicist, which we discuss further in part B.

Nadya, who was also interviewed during her last semester in the Physics program, interpreted the question slightly differently. Rather than talking about access to the field or the ambiguities that surround the idea of physicist, she talked about the stereotypes that previously prevented her from seeing herself as a physicist. She stated that she had often had difficulties in identifying as a physicist because of the prevalent stereotypes of men in physics: "I don't know, I could never, I never saw myself being able to be like that, because physicists are like- They're like big smart men that know everything. And I'm like, 'Oh my God, that's not me.' (Laughs)." This wasn't the first or last time Nadya mentioned the stereotype of a male physicist in her interview. She expressed that she was aware of what people think of when they hear the word physicist and that she was fully aware that she would never fit into the stereotype. However, we will see in section B that despite being aware of the stereotype, her personal definition of a physicist is totally different.

\section{B. Physics as a Practice}

It was through Paige's personal conception of what it means to be a physicist that the authors began seeing a clear connection between recognition and practicing physics. Paige, who was conducting Ph.D. research at the time of her interview, had received both her bachelor's and master's degrees in Physics from Texas State. When the interviewer asked "Can you describe a physicist?" Paige responded immediately: "Okay. Well my idea of a physicist is just someone who does physics...If you're doing science, you're a fucking scientist. The end." She went on to narrow down the previous statement slightly: "I do think that there is some merit in being a little exclusive with that term.... When it comes to policy procedure though I do think that you need some motherfucking credentials."

After analyzing Paige's narrative, we began examining the other interviews to see if they shared similar ideas in their understanding of physicists. While no one said the exact same words, the research team interprets the women's definitions in a similar way to Paige's: the women described a physicist as someone who questions the world they're in, someone who actively identifies as a physicist, and, again, someone who is doing physics. Frankie's answer was most similar to Paige's:

"You have to keep telling yourself, 'this is what you're doing. You're good at this. You're a physicist.' And like, Yay! It was a lot of 'We're doing this!' When I got to that point when I was like I've come too far to turn around now."

Nadya's initial response to the question read like a word association game. Physics equates to...: "Honestly, like, just Math. I think of Math. I think of endless, endless Math. Like I just- I just think of Math..." Nadya then began describing certain characteristics of what a physicist does in their practice: "...I think of like people who really questions the world that they live in. Like people who are trying to get to a deeper understanding of where we live and whatnot."

Autumn also expressed similar ideas to Nadya's. Her description was based on characteristics and personal ownership of the title physicist. She told the story of the moment she first identified as a physicist:

"It's almost like: as long as your mind is like, 'This is who I identify as' or 'who I want to be.' I feel like that one day in my first astronomy physics orientated class 
when I was like, 'I want to be a physicist.'- I think that was the moment when I became a physicist. That inspired, 'this is what I want to be.",

Autumn's interpretation of a physicist as someone who selfidentifies as such is immensely important to the narrative she paints for herself. The practice of owning the identity and the title of physicist inspired her to pursue the degree.

Based on the participants' description of physicist as someone who self-identifies as such, the research team believes that this understanding lead to their ability not only to see themselves as physicists but to recognize their peers as well.

\section{Recognition of self and others as physicists}

After asking for their description of a physicist, the interviewer asked all participants "Do you see yourself as a physicist?" and "Do others see you as a physicist?" All women reported self-recognition and ownership of their physics identity, and believed their professors, peers, and others saw them as physicists. While analyzing the video data, the research team noticed that all of the participants talked unprompted about the students they engaged with both in and out of class, and described recognizing them as physicists, despite there being no interview question that asked "Do you see others as physicists?" Nadya replied with hope that because she sees her peers as physicists they would see others as the same:

"I would- I would hope that everybody would see me and each other as equals and as physicists and altogether, and that we're all trying to get through this...yeah. So, I would really hope, because I think we're all- we're all... I mean, I know for sure I see all my equals as physicists because we all out here. We all have something to bring to the table, we all have our strengths, we all have our weaknesses, and we're all trying to make it through."

Frankie's statement on her peers was very similar. She recognized her friends and herself as physicists: "But, yeah, I really think a lot of it is, I have friends that are physicists. I'm a physicist. We're all physicists."

The research team related the women's interpretations of physicist to how they recognized others as physicists. Because there was no strict definition, no need for credentials, but only a relation to physics as a practice they and their peers participated in, they were able to apply the title physicist to others and to themselves without exclusion.

\section{DISCUSSION AND CONCLUSIONS}

The purpose of this study is to understand how these women think about the identity physicist and how this relates to their own physics identity. The current analysis demonstrates that the participants in this study related strongly to the idea that physicists are those who participate in the practices of physics. This concept of physicist avoids the cultural bias exemplified in the broader culture's idolizing of Einstein and media portrayal of Sheldon. These interviews also demonstrate the critical importance of personally identifying as a physicist in terms of empowerment and belonging.

An emphasis by departments and faculty on the idea of physics as something people do, rather than physics as a set of knowledge already created by inaccessible geniuses, can support access for marginalized students to the identity of physicist. This is consistent with previous research illustrating the importance of recognition as a component of the negotiated experience of identity within a community of practice $[3-5,10]$. Recognition as a "physics person" by important others is critical to the development of positive physics identity [4]; framing physics as a set of practices broadens the range of activities that can be recognized by peers and mentors as legitimate and productive participation as physicists.

Each of the participants in the study was aware of and acknowledged the cultural image of physicists as white, cisgender, heterosexual male geniuses. Despite this, they were able to reject the common stereotype in favor of an image fully accessible to them: that of a person engaged in the practices of physics, alongside others, drawing on each other's strengths and supporting each other as equals.

In future work we plan to interview LGBTQ+ women and women of color in physics majors at other institutions in order to compare their experiences with those of our participants in the Texas State physics department.

\section{ACKNOWLEDGMENTS}

The authors are grateful to the women who agreed to participate in interviews in support of our research. This work has been supported in part by NSF grants DUE1557405, DUE-1557276, and PHY-0808790.
[1] Ong, M., Wright, C., Espinosa, L., \& Orfield, G. (2011). Inside the Double Bind: A Synthesis of Empirical Research on Undergraduate and Graduate Women of Color in Science, Technology, Engineering, and Mathematics. Harvard Educational Review, $81(2)$,

https://doi.org/10.17763/haer.81.2.t022245n7x4752v2

[2] T. Atherton et al., LGBT Climate in Physics: Building an Inclusive Community (Am. Phys. Society, College Park, MD, 2016).

[3] Hyater-Adams, S., Fracchiolla, C., Finkelstein, N., \& Hinko, K. (2018). Critical look at physics identity: An operationalized framework for examining race and physics identity. Physical Review Physics Education Research, 14(1), 10132. https://doi.org/10.1103/PhysRevPhysEducRes.14.010132 
[4] Hazari, Z., Sonnert, G., Sadler, P. M., \& Shanahan, M.-C. (2010). Connecting high school physics experiences, outcome expectations, physics identity, and physics career choice: A gender study. Journal of Research in Science Teaching, 47(8), n/a-n/a. https://doi.org/10.1002/tea.20363

[5] Wenger, E. (1998). Communities of Practice: Learning, Meaning, and Identity. Systems Thinker. https://doi.org/10.2277/0521663636

[6] Crenshaw (Black Feminist Theory) Jaggar, A. M., \& Crenshaw, K. (1994). Demarginalizing the Intersection of Race and Sex: A Black Feminist Critique of Antidiscrimination Doctrine, Feminist Theory, and Antiracist Policies, 1989(1), 3952.

[7] Ong, M. (2005). Body Projects of Young Women of Color in Physics: Intersections of Gender, Race, and Science. Social Problems, 52(4), 593-617.

https://doi.org/10.1525/sp.2005.52.4.593

[8] Cech, E. A., \& Waidzunas, T. J. (2011). Navigating the heteronormativity of engineering: The experiences of lesbian, gay, and bisexual students. Engineering Studies, 3(1), 1-24.

https://doi.org/10.1080/19378629.2010.545065

[9] Cooper, K. M., \& Brownell, S. E. (2016). Coming out in class: Challenges and benefits of active learning in a biology classroom for LGBTQIA students. CBE Life Sciences Education, 15(3), 119. https://doi.org/10.1187/cbe.16-01-007

[10] Close, E. W., Conn, J., \& Close, H. G. (2016). Becoming physics people: Development of integrated physics identity through the Learning Assistant experience. Physical Review Physics Education Research, 12(1), 010109.

https://doi.org/10.1103/PhysRevPhysEducRes.12.010109

[11] APS Education and Diversity Committee, and IPEDS APS graphs

[12] Gasman, M., \& Conrad, C. F. (2013). Educating All

Students: Minority Serving Institutions. Penn Center for Minority Serving Institutions, Retrieved

from http://repository.upenn.edu/gse pubs/339

[13] TXST Enrollment Explorer. (n.d.)

https://www.ir.txstate.edu/ir-self-service/self-service

Retrieved 06/27/2019

[14] National Science Foundation, National Center for Science and Engineering Statistics. 2019. Women, Minorities, and Persons with Disabilities in Science and Engineering: 2019. Special Report NSF 19-304. NSF graphs

[15] Williams Crenshaw, K. (1994) Mapping the Margins: Intersectionality, Identity Politics, and Violence Against Women of Color. 